\title{
A Novel Broadband Ultrasonic Location System
}

\author{
Mike Hazas ${ }^{1}$ and Andy Ward ${ }^{2}$ \\ ${ }^{1}$ Laboratory for Communications Engineering \\ University of Cambridge, United Kingdom \\ moh29ecam.ac.uk \\ 2 awardecantab. net
}

\begin{abstract}
Indoor ultrasonic location systems provide fine-grained position data to ubiquitous computing applications. However, the ultrasonic location systems previously developed utilize narrowband transducers, and thus perform poorly in the presence of noise and are constrained by the fact that signal collisions must be avoided. In this paper, we present a novel ultrasonic location system which utilizes broadband transducers. We describe the transmitter and receiver hardware, and characterize the ultrasonic channel bandwidth. The system has been deployed as a polled, centralized location system in an office. Test results demonstrate that the system can function in high levels of environmental noise, and that it has the capability for higher update rates than previous ultrasonic location systems.
\end{abstract}

\section{Introduction}

Ubiquitous computing applications require context information in order to blend seamlessly into the environment and to unobtrusively aid people in their everyday lives. A particularly important component of context is the location of people and the objects they interact with. Numerous ubiquitous applications utilizing location information have been developed [1].

Various methods exist for determining the location of a person or object. All involve gathering data by sensing a real-world physical quantity, and using it to calculate or infer a position estimate. The data can be gathered via a number of physical media; previously developed location systems have used infrared light [2, 3, 4], visible light $[5,6]$, ultrasound $[7,8,9]$, and wireless LAN-based radio [10, 11].

The desirable property of ultrasonic location systems is that they have the capability to be fine-grained, meaning they can estimate location with a high degree of resolution. This is because the speed of ultrasound in air is sufficiently slow to allow the time-offlight of a signal to be accurately measured between a fixed unit in the environment and a mobile unit functioning as a tag on a person or object. In the past, fine-grained ultrasonic location data has been used for context-aware applications including mobile desktop control [12], 3D mice and virtual buttons [13], and augmented reality [7]. However, previous ultrasonic location systems have employed narrowband signals. This makes them susceptible to noise, and their update rate is limited due to the fact that signal collisions must be avoided.

This paper presents a novel location system utilizing broadband ultrasound. First, we review previous narrowband ultrasonic location systems, and highlight the advantages a broadband system would offer. We then describe the prototype transmitter and

In Proceedings of UbiComp 2002: Fourth International Conference on Ubiquitous Computing, Lecture Notes in Computer Science volume 2498, pages 264-280, Göteborg, Sweden, September 2002. (C) Springer-Verlag. 
receiver hardware, collectively referred to as Dolphin, which incorporate a broadband ultrasonic transducer new to the field of ubiquitous computing. We detail the implementation of a polled, centralized location system using Dolphin, and report the measurements of its location accuracy. Finally, we discuss our ongoing work with the Dolphin prototypes.

\section{Related Work}

There are a number of ultrasonic location systems presented in the literature. The Constellation system [7] tracks a mobile unit consisting of a 3D inertial sensor and a number of ultrasonic sensors. Location is calculated using time-of-flight measurements between the mobile unit and fixed transmitters in the environment. An accuracy of approximately $5 \mathrm{~mm}$ is reported, but the mobile tracking unit, worn on the head and belt of a user, is too obtrusive for the goals of ubiquitous computing.

In the Bat system [8, 13, 14], users wear small badges which emit an ultrasonic pulse when radio-triggered by a central system. The system determines pulse times-offlight from the badges to a network of receivers on the ceiling, and calculates the $3 \mathrm{D}$ positions of the badges using a multilateration algorithm. The system yields location information with an accuracy of approximately $3 \mathrm{~cm}$.

The Cricket location system [9, 15] consists of independent, unconnected beacons distributed throughout a building. The beacons send an RF signal while simultaneously sending an ultrasonic pulse. Small devices called listeners, carried by users, infer their locations using time-of-flight methods, with accuracies on the order of $6 \mathrm{~cm}$ in the best cases. The beacons broadcast at random times in order to minimize signal collisions. The listeners can independently locate themselves, avoiding the compromises of user privacy involved with centralized systems.

A similar approach [16] uses four ultrasonic transducers placed at the corners of a square on the ceiling, and wired to a controller. The controller sends an RF trigger, and then issues a pulse from each of the four transmitters in succession. A mobile receiver unit connected to a handheld computer receives the pulses, and estimates its location with accuracies between 10 and $25 \mathrm{~cm}$.

\section{Motivation}

The above four systems share one common trait: they utilize narrowband ultrasonic transducers for their ranging measurements. Generally speaking, narrowband systems have two inherent disadvantages.

1. Single-user access - if multiple co-located transmitters send at the same time, their signals can interfere with one another, and make it difficult for the receiver to distinguish between them.

2. Poor performance in the presence of in-band noise. 
The systems described above avoid the first problem by attempting to ensure that co-located transmitters send their signals one-at-a-time. ${ }^{3}$ This solution comes at the expense of a slower update rate; allowing only one transmitter to send at a time constrains the number of location updates possible for a given time interval.

The second problem, however, has not been addressed. People create ultrasonic noise through their everyday actions, although they are rarely aware of it because ultrasound is inaudible. Examples include the clink of a pencil dropping onto a desk, the clacking of someone typing on a computer keyboard, or the rustling of a bag of potato chips being opened. These kinds of sounds occur frequently in typical indoor environments. For the duration of such an occurrence, existing ultrasonic location systems are prohibited from generating accurate, up-to-date position estimates for mobile units co-located with the noise source.

We propose that broadband spread spectrum techniques be used to overcome these two limitations, facilitating multiple-access location systems which are robust in the presence of noise. The aim of the Dolphin prototype is to assess the practical applications of such systems.

\section{Hardware Design}

This section describes the design and properties of the Dolphin transmitter and receiver prototype hardware. The units were designed to facilitate flexibility, in order to fully gauge the potential of this new technology. This meant tethering the units to a power supply and a workstation PC for signal synthesis and analysis. However, making the units into small, light, wireless, and battery-powered mobile tags is feasible, and the modifications needed in order to accomplish this are set out in Sect. 4.5. The prototype system was not designed to operate outside of the frequency range of 20 to $100 \mathrm{kHz}$. Sounds below $20 \mathrm{kHz}$ are perceivable by humans, and the use of frequencies above $100 \mathrm{kHz}$ is limited by the attenuation of ultrasound in air [17].

\subsection{Transducer Selection}

The narrowband transducers used in existing ultrasonic location systems rely on piezoelectric ${ }^{4}$ ceramics as their active elements. These kinds of transducers are inexpensive, small, rugged, and have a high sensitivity. However, they are highly resonant, and in most cases have a usable bandwidth of less than $5 \mathrm{kHz}$.

Electrostatic transducers, on the other hand, possess high sensitivity and extremely wide bandwidth. However, they are not very rugged, and are expensive, making their deployment on a large scale prohibitive.

\footnotetext{
${ }^{3}$ An exception to this is the Bat system, which uses a differential-phase modulation technique, allowing up to three tags to transmit simultaneously [13].

${ }^{4}$ Broadly speaking, a piezoelectric material is one in which a mechanical change of the material is proportional to a change in the electric field across the material. Thus, when a piezoelectric material is subjected to a physical force (such as pushing, pulling, bending or twisting) a charge is generated across it, and vice versa.
} 
Certain synthetic polymer films have piezoelectric properties [18], and are known as piezopolymer films or simply piezo films. These piezo films, most notably polyvinylidene fluoride (PVDF), have been applied as ultrasonic transducers in hydrophone applications, and for high frequency (greater than $200 \mathrm{kHz}$ ) medical imaging and nondestructive testing $[19,20,21,22]$. Piezo film transducers are small, inexpensive, more rugged than electrostatic transducers, and have a wide frequency bandwidth. However, they have low sensitivity. This means that as transmitters they must be driven with high voltages, and as receivers they are particularly sensitive to noise.

A piezo film ultrasonic transducer for air ranging has been designed by Fiorillo [23, 24] and further characterized by Wang and Toda [25]. It consists of a small, rectangular piece of piezo film mounted along two of its edges to form a half-cylinder. As depicted in Fig. 1, the design works by virtue of the fact that the ends of the piezo film are firmly clamped. Any change in the radius $r$ of the hemicylinder can be seen as a change in the length $l$ of the piezo film, since $l=\pi r$. Due to the piezoelectric properties of the film, changes in $l$ are approximately proportional to the voltage across the thickness of the film. Thus, the clamped piezopolymer can function in two ways:

1. As a transmitter, when voltages are applied to it, and

2. As a receiver, when airborne acoustic waves impact it.

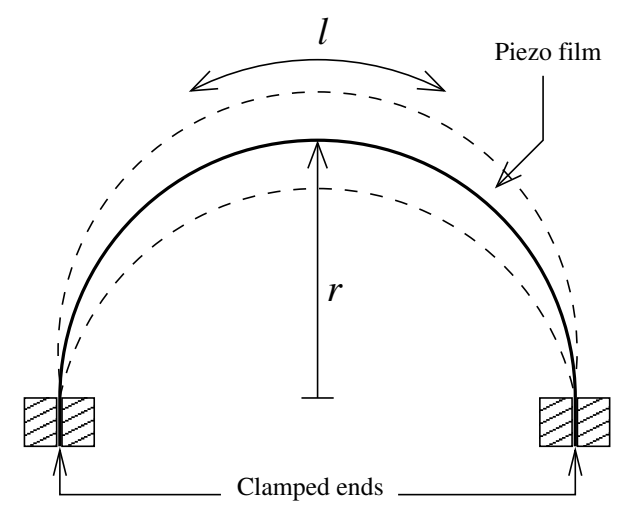

Fig. 1. Operation of a clamped cylindrical piezopolymer transducer [24]

This transducer was designed to measure short distances, and has a maximum range of $40 \mathrm{~cm}$. The construction and interfacing of piezo film ultrasonic transducers for air ranging over distances of $1.5 \mathrm{~m}$ or more have not been presented previously. We have applied a modified version of Fiorillo's design in the Dolphin prototype transmitters and receivers, suitable for air ranging over room-scale distances. 


\subsection{Transmitter}

As shown by Fig. 2, the transmitter is designed to convert an electrical voltage signal into an ultrasonic one, using a power op amp with some signal conditioning, a step-up transformer, and a piezo film transducer. A Dolphin transmitter unit is shown in Fig. 3, and measures $60 \mathrm{~mm} \times 94 \mathrm{~mm} \times 15 \mathrm{~mm}$.

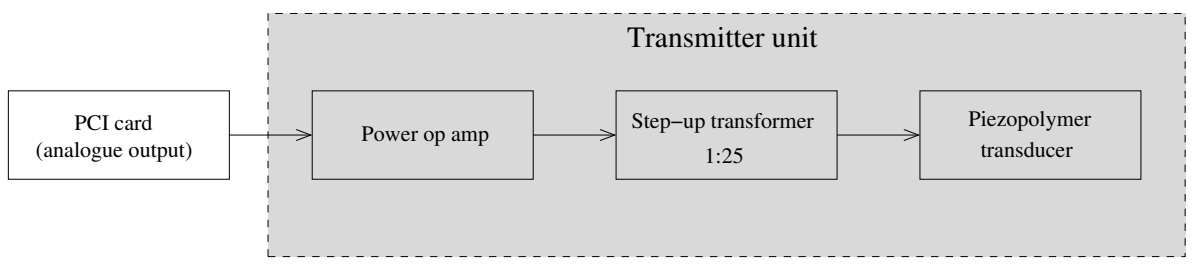

Fig. 2. Transmitter block diagram

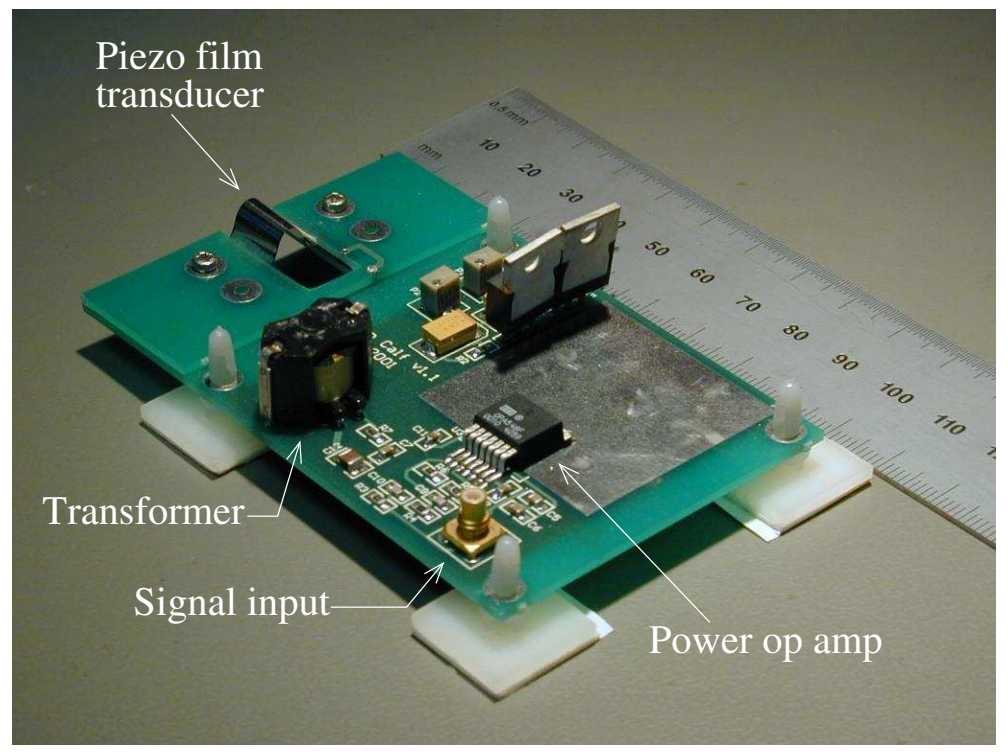

Fig. 3. Dolphin transmitter 
Signal Synthesis. The signals for the transmitters were synthesized digitally on a workstation PC, to allow the structure of the ranging message to be changed easily in software. The signals were sent out through a PCI card which performs digital-to-analogue conversion, and carried to the transmitter units via coaxial cable.

Electronic Interface. It was desired that the transmitter unit be capable of driving its piezo film transducer with signal levels ranging from tens to hundreds of volts peak-topeak, in order to provide flexibility. Thus, it was necessary to use a power op amp in conjunction with a step-up transformer to drive the piezo film transducers. The transformer has a voltage gain factor of 25 in the frequency range of $20 \mathrm{kHz}$ to $100 \mathrm{kHz}$. The high voltage side of the transformer is connected directly to the piezo film transducer.

\subsection{Receiver}

Figure 4 depicts the architecture of the receiver. Ultrasound travelling through the air is converted by the piezo film transducer into an electrical signal which is buffered by a charge amplifier. The signal then undergoes minor conditioning before being fed into data acquisition PCI cards at a workstation. The receiver unit, shown in Fig. 5, measures $60 \mathrm{~mm} \times 115 \mathrm{~mm} \times 75 \mathrm{~mm}$.

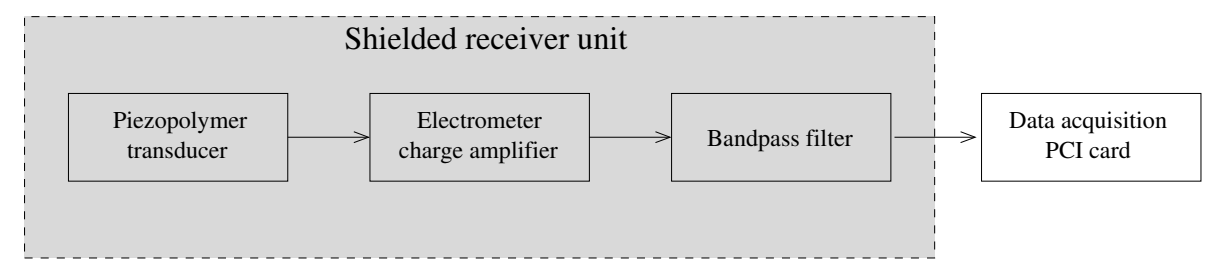

Fig. 4. Receiver block diagram

Electronic Interface. The piezo film is directly connected to an electrometer ${ }^{5}$ in a charge amplifier configuration. The charge amplifier produces a voltage proportional to the charge difference between its input terminals. This preamplified signal is then passed through a filter which removes frequencies outside the desired operating band of the system.

Shielding and Low-Noise Requirements. Because of the high voltages produced by the transmitter unit and the extremely sensitive nature of the receiver, care had to be taken to reduce electromagnetic interference as much as possible. In early experiments,

\footnotetext{
${ }^{5}$ An electrometer is a low-noise, high impedance op amp specifically designed to interface with sensors.
} 


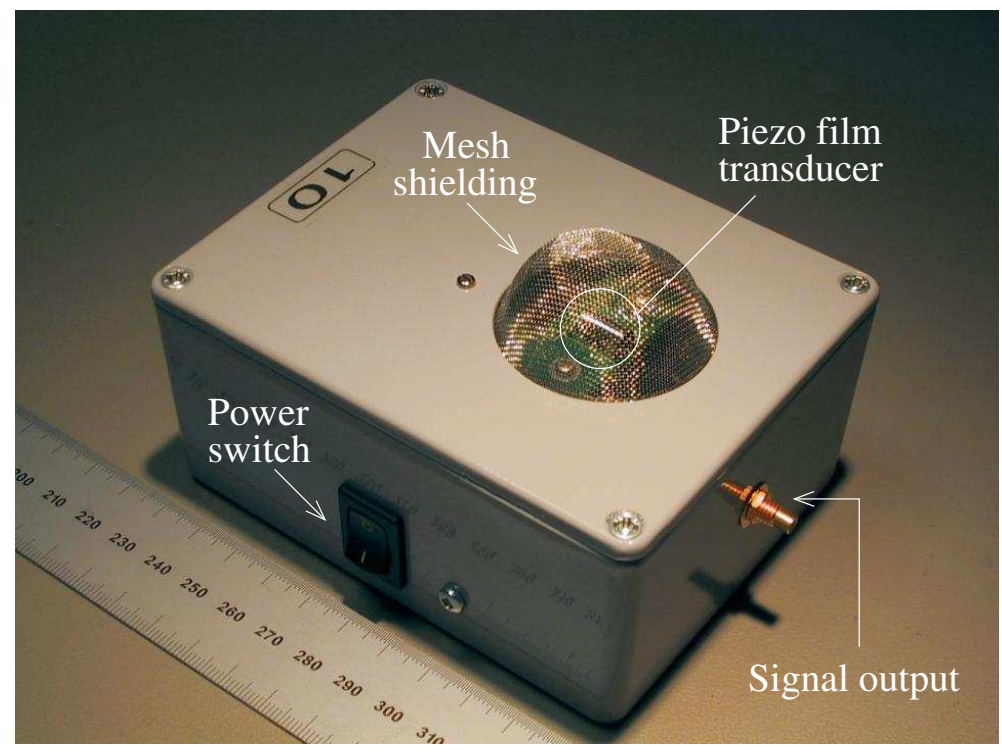

Fig. 5. Dolphin receiver

it was found that the noise at the receiver due to mains interference and electromagnetic pickup from the transmitter was much greater than the signal converted by the piezo film as a result of airborne ultrasound. Several measures were taken to reduce noise at the receiver to an acceptable level.

1. A physically short, low impedance connection between the transducer and the electrometer input reduces the risk of signal degradation.

2. The receiver unit's circuitboard is mounted in a metal case connected to the power ground of the receiver circuit, and the receiving transducer is shielded using a metal mesh dome.

3. Two standard $9 \mathrm{~V}$ batteries power the receiver unit, and consideration was taken in the physical layout of the receiver circuitboard, to minimize power supply interference.

Data Acquisition. Using coaxial cable, the signal outputs of the receiver units were connected to data acquisition cards at a workstation PC. The PCI cards perform analogue-to-digital conversion. A sampling rate of $208 \mathrm{kHz}$ (over twice the highest frequency of interest) was chosen for each receiver. The converted signals are then processed using software running on the workstation PC. This method allows flexibility when conducting experiments with the Dolphin prototype, as signals can be analysed in real time using a variety of methods, implemented as $\mathrm{C}++$ software. 


\subsection{Channel Bandwidth Capabilities}

The bandwidth of the ultrasonic channel was characterized by measuring the frequency response of five transmitter-receiver pairs in an anechoic chamber. In the experiments, the transmitter was directly facing the receiver at a distance of one metre. Figure 6 shows the averaged channel frequency response in decibels, referenced to the receiver voltage noise floor.

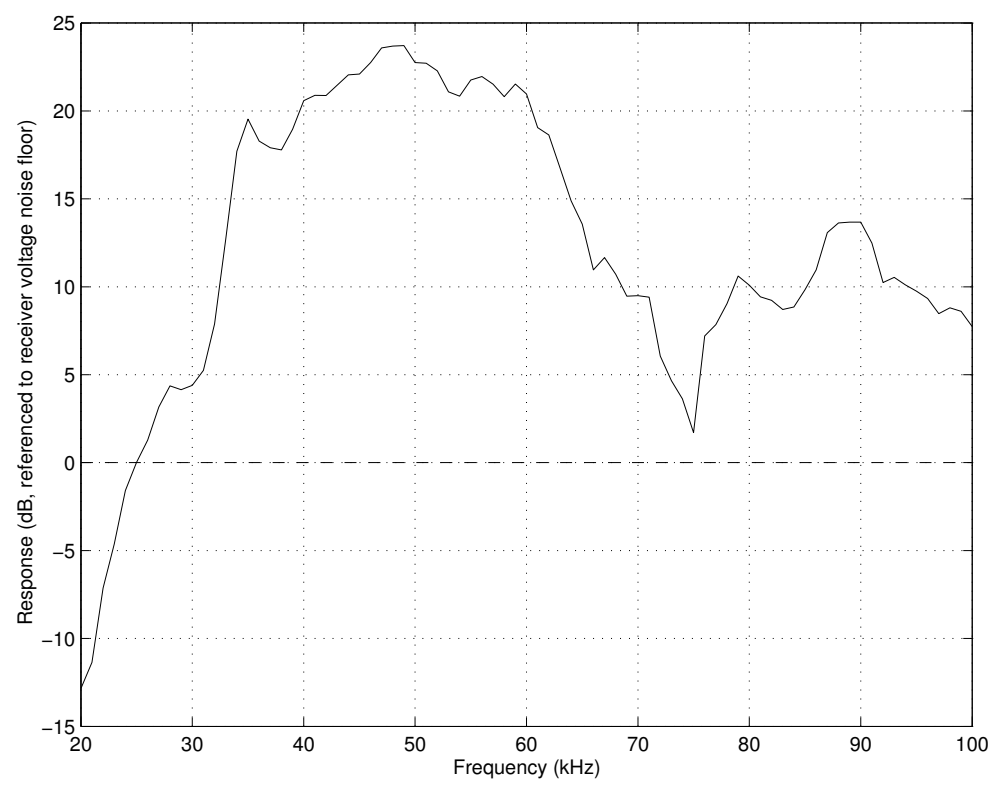

Fig. 6. Ultrasonic channel bandwidth at one metre (receiver noise floor shown as a dashed line)

For on-axis communications at a distance of one metre, the channel has $76 \mathrm{kHz}$ of bandwidth above the noise floor. This is wide enough to justify the use of the Dolphin prototype to explore the capabilities of broadband ultrasonic location systems. As shown by Table 1, if the signal is attenuated by off-axis transmission or greater distances between the transmitter and receiver, there still remains bandwidth above the noise floor.

\subsection{Modifications Needed for a Production System}

The transmitter's power op amp was chosen to make the prototype as flexible as possible, in keeping with the system design goals. The power drawn by the unit when driven with typical signal levels measured over $1 \mathrm{~W}$. Clearly, this is unacceptable for a mobile device. However, the power op amp is inefficient, and the actual currents required to drive the transducer are much lower. Specialized op amps exist, such as those used to 
Table 1. Effect of signal attenuation on bandwidth above noise floor

\begin{tabular}{||c|c|c||}
\hline $\begin{array}{c}\text { Signal } \\
\text { attenuation }(\mathrm{dB})\end{array}$ & $\begin{array}{c}\text { Equivalent } \\
\text { distance }(\mathrm{m})\end{array}$ & $\begin{array}{c}\text { Bandwidth above } \\
\text { noise floor }(\mathrm{kHz})\end{array}$ \\
\hline 0 & 1.00 & 76 \\
5 & 1.78 & 67 \\
10 & 3.16 & 47 \\
15 & 5.62 & 30 \\
\hline
\end{tabular}

drive cellular phone earpiece speakers, which can supply sufficient currents and also have a low-power shutdown mode. If the transmitter were to be redesigned with one of these, power consumption during transmission could be decreased to below $500 \mathrm{~mW}$, and virtually eliminated outside of transmission times.

Both the transmitter and the receiver are too large and bulky to be used as mobile tags or unobtrusively installed in the environment. Before being deployed for everyday use, the circuitboards would be redesigned, and parts chosen which are more appropriate for small, mobile devices. If the units were deployed as mobile tags, components could be chosen which have sufficiently low operating voltages so that they could be powered by a single-cell battery.

\section{Implementing a Polled, Centralized Location System Using Dolphin Prototypes}

In order to explore the concepts of multiple access and noise immunity, the Dolphin transmitter and receiver units were used to implement a polled, centralized ultrasonic location system in an office. ${ }^{6}$ This section describes the implementation, reports the results of measurements, and discusses those results.

\subsection{Test System Configuration}

The system was installed in an office $3.5 \mathrm{~m} \times 2.6 \mathrm{~m}$ in size, with a ceiling height of $2.3 \mathrm{~m}$. Eight receivers were affixed to the ceiling (Fig. 7), such that their transducers pointed directly down at the floor.

A Dolphin transmitter, designated $\mathrm{T}_{\text {mobile }}$, was placed at four different heights $(0.10$, $0.50,1.0$, and $1.40 \mathrm{~m}$ above the floor), at each of sixteen points on a grid, shown by the "•" marks in Fig. 8. Four additional transmitters were placed in fixed positions near the walls of the room, at a height of approximately $0.75 \mathrm{~m}$ above the floor, with their transducers pointing upwards. These fixed transmitters were triggered to send simultaneously with $\mathrm{T}_{\text {mobile }}$ in order to test the multiple-access potential of the broadband system.

\footnotetext{
${ }^{6}$ Polled indicates that transmitters are told when to send their ranging messages, and centralized means that there is a central service which collects the time-of-flight data and uses it to estimate the locations of mobile tags. The Bat is an example of a polled, centralized location system.
} 


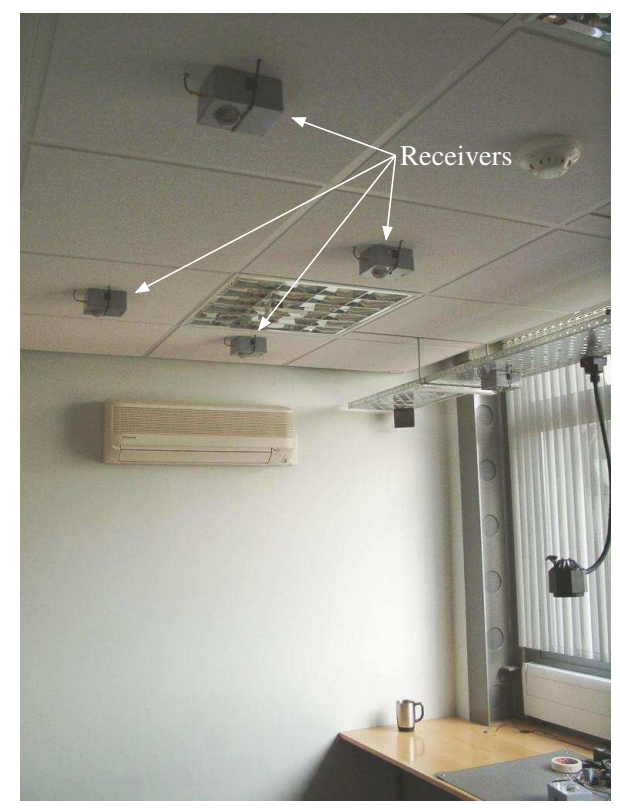

Fig. 7. Room with prototype broadband ultrasonic location system installed

In-band ultrasonic noise was generated by using a motor to jingle keys attached to a string. This type of noise has been known to make narrowband ultrasonic location systems fail to report location estimates altogether. The ultrasonic sound pressure level generated by the jingling keys was approximately $67 \mathrm{~dB}$ at the ceiling. ${ }^{7}$

\subsection{Ranging Message Signal Structure}

A direct sequence spread spectrum (DSSS) signal structure was applied in order to achieve multiple-access properties and robustness towards noise. In a DSSS system, a data-modulated carrier signal is further modulated by a pseudo-random binary sequence [26]. The rate at which the pseudo-random sequence is applied is usually much faster than the data rate. This has the effect of spreading the spectrum of the signal. One can think of this as distributing the information content (i.e. data) over a large range of frequencies, instead of centring it closely about the carrier frequency. Spreading the spectrum in this way makes the signal more robust in the presence of in-band noise.

Gold codes are a particular set of pseudo-random sequences which have low autoand cross-correlation properties [27]. If different Gold codes are assigned to users in a DSSS system, their signals can be sent simultaneously and still be separated at the receiver. This gives the system multiple-access properties, since corruption due to signal collision is minimal.

\footnotetext{
${ }^{7}$ This decibel figure is referenced to an RMS sound pressure level of $20 \mu \mathrm{Pa}$, and was measured by a precision ultrasonic microphone.
} 


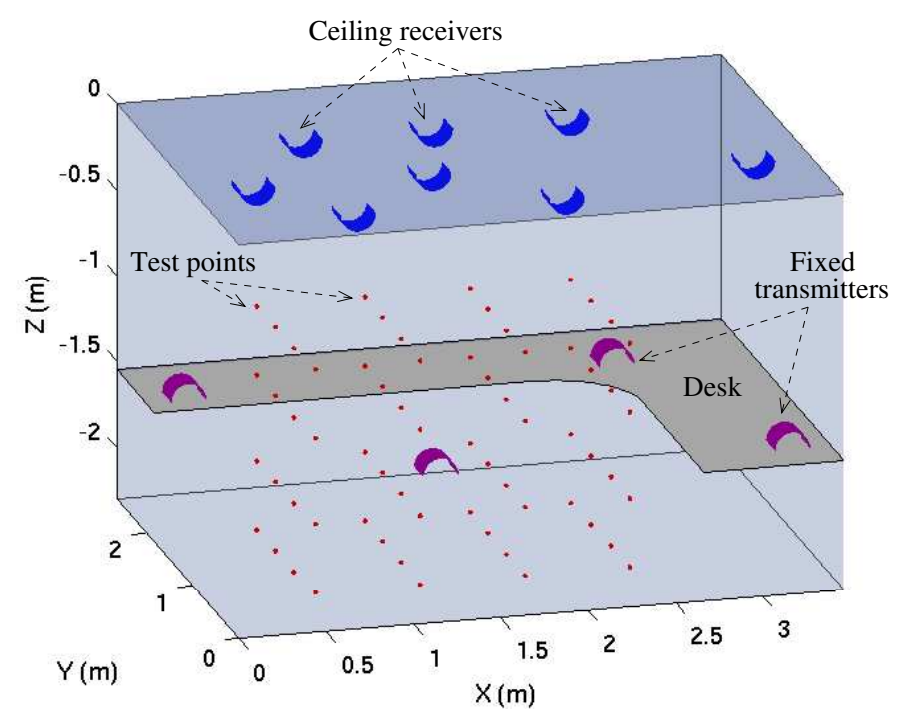

(a) $3 \mathrm{D}$ view

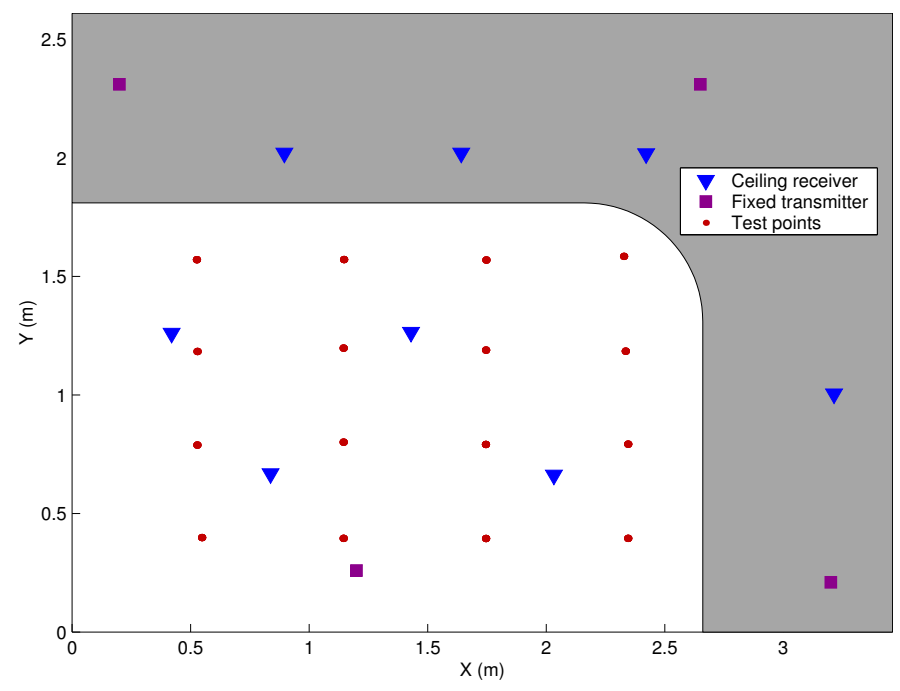

(b) Top view

Fig. 8. Room test setup 
Gold codes are employed in the outdoor Global Positioning System (GPS), and Girod and Estrin have used codes with similar properties in their acoustic ranging system, which operates in the audio frequency range [28]. What makes the Dolphin prototype unique and practical for ubiquitous computing is that it enables the use of spread spectrum ultrasonic signals. This allows the creation of robust, fine-grained location systems which are unobtrusive (i.e. inaudible).

The ranging messages used in the measurements presented below consisted of a $50 \mathrm{kHz}$ carrier wave, modulated by a Gold code using binary phase-shift keying. ${ }^{8}$ The Gold code had a length of 511 bits, and was applied at a rate of $20 \mathrm{kHz}$, giving a ranging message duration of about $25 \mathrm{~ms}$. Figure 9 shows the frequency spreading effect when a Gold code is used to modulate the carrier.

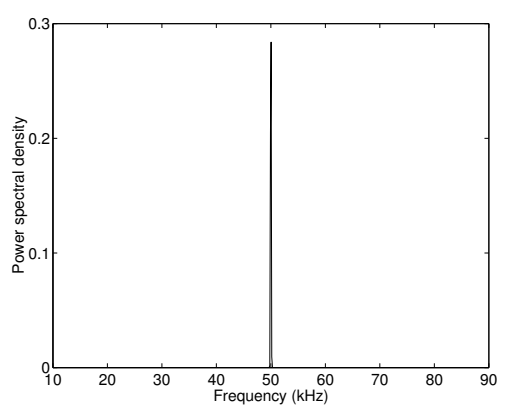

(a) $50 \mathrm{kHz}$ carrier only

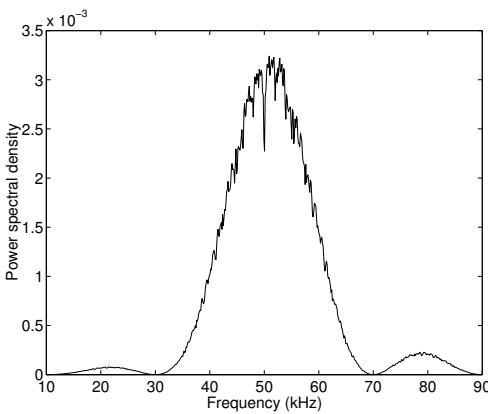

(b) $50 \mathrm{kHz}$ carrier spread by $20 \mathrm{kHz}$ Gold code

Fig. 9. Spreading the spectrum of the ranging message

\subsection{Location Estimation}

The location of $\mathrm{T}_{\text {mobile }}$ was estimated using the following steps.

1. The times-of-flight for the $T_{\text {mobile }}$ ranging messages were measured by correlating the receivers' signals against the expected signal (a $50 \mathrm{kHz}$ carrier modulated by $\mathrm{T}_{\text {mobile }}$ 's Gold code). The time-of-flight was defined as the time between the triggering of $\mathrm{T}_{\text {mobile }}$ and the occurrence of a large peak in the correlated data.

2. The times-of-flight were converted to transmitter-to-receiver distances using the speed of sound in air.

3. Using the accurately surveyed positions of the receivers and the transmitter-toreceiver distances, location was estimated by employing the multilateration algorithm described by Ward [14].

\footnotetext{
${ }^{8}$ The carrier was not modulated by any data.
} 
Since the receivers are coplanar, a minimum of three distances is needed to estimate the transmitter's position, with the assumption that it is below the ceiling. However, we required that at least four distances be measured, enabling the multilateration algorithm to estimate the error of its produced locations. The algorithm does not return a position estimate if there are fewer than four transmitter-to-receiver distances, or if the distances have a high error estimate.

\subsection{Results}

Five hundred location readings for $\mathrm{T}_{\text {mobile }}$ were taken at each of the sixty-four test points under three test conditions:

1. $T_{\text {mobile }}$ transmitting by itself,

2. $T_{\text {mobile }}$ and the four fixed units transmitting simultaneously, and

3. $\mathrm{T}_{\text {mobile }}$ and the four fixed units transmitting simultaneously in the presence of noise (i.e. jingling keys).

Figure 10 shows the error distribution functions of the returned locations for the three test conditions. Table 2 indicates the percentage of readings for which the algorithm returned a location estimate. The $95 \%$ confidence levels of the 3D position accuracies are also listed.

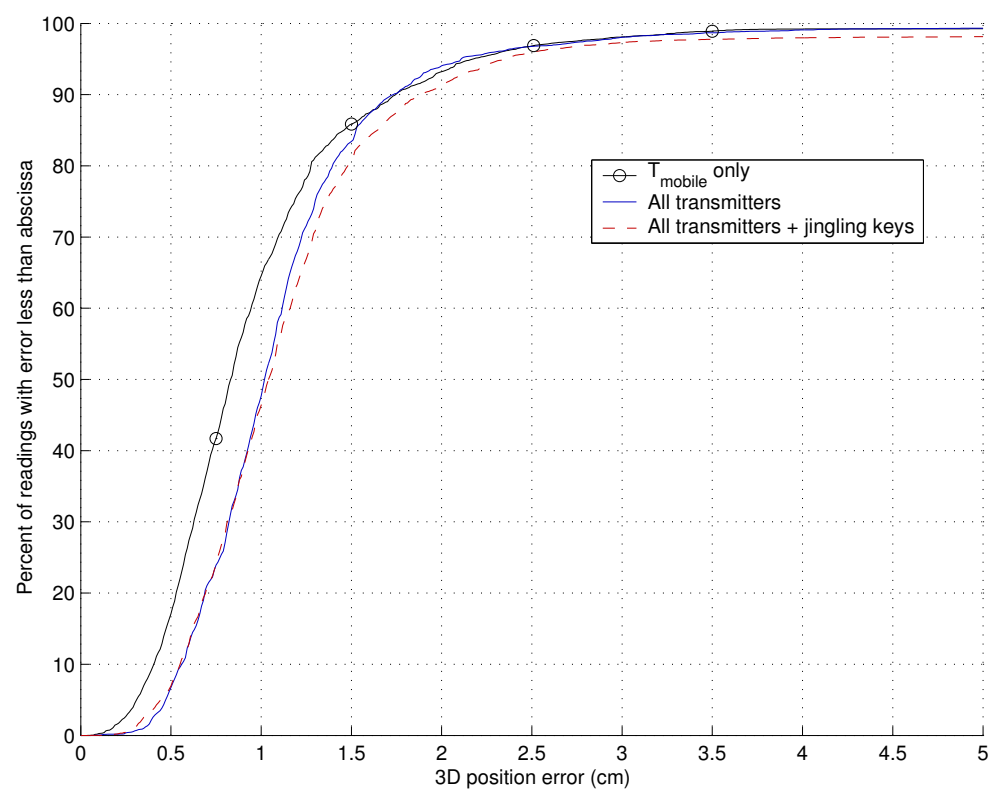

Fig. 10. Accuracy of location estimates for $T_{\text {mobile }}$ 
Table 2. Location system performance

\begin{tabular}{||c|c|c||}
\hline $\begin{array}{c}\text { Test } \\
\text { condition }\end{array}$ & $\begin{array}{c}\text { Readings } \\
\text { returned }(\%)\end{array}$ & $\begin{array}{c}95 \% \text { confidence } \\
\text { level }(\mathrm{cm})\end{array}$ \\
\hline 1 & 93 & 2.17 \\
2 & 75 & 2.11 \\
3 & 68 & 2.34 \\
\hline
\end{tabular}

The update rate of the algorithm was approximately five location estimates per second. This limit is imposed by the computational power of the workstation PC, as it has to correlate against all of the incoming receiver signals. It is important to note that with a broadband ultrasonic location system such as this one, the main factor limiting the update rate is computational power. This represents a departure from narrowband systems, where the limiting factor is the need to allocate a time slot to each transmitter, providing sufficient time for reflections to die down between transmissions.

However, in a system deployed for everyday use, hardware correlators could be used to produce time-of-flight data in approximately real time. This would facilitate an update rate appropriate for mobile desktop control and virtual button applications. However, because of the multiple-access nature of the system, the location updates are simultaneous, giving the system a superior performance in applications which require both high update rate and low latency between updates of different objects. Examples include 3D mice, gesture recognition, augmented and virtual reality, and the simultaneous tracking of moving people and the devices they carry.

\section{Current Work}

A polled, centralized architecture is not the only way to implement a location system using Dolphin. It is also possible to create an anonymous system, where mobile units estimate their position, instead of depending on a central service. In such a system the fixed units in the environment could function as transmitters, and the mobile units as receivers. A main advantage of this kind of system is that it maintains user privacy; an example of an anonymous location system is Cricket.

However, Dolphin's broadband properties can be applied to gain improved performance. Using a spread spectrum multiple access technique would not only increase robustness toward noise, but the transmitters could also send simultaneously and much more often, since there would be no need to avoid signal collisions. Work on an anonymous broadband system is underway. Dolphin transmitters have been installed in the ceiling of an office, and the software to calculate the positions of mobile receivers is being developed.

Another research avenue involves transmitting small amounts of data across the ultrasonic channel. Such data transmission would be useful where no infrared or radio link exists between the mobile and fixed units. Note that the communication would only be one-way, unless the fixed and/or mobile units were equipped with ultrasonic transceivers. Such a device would be feasible to implement; the transducers used in 
the transmitter and receiver units are physically similar, making it possible to combine the interface electronics into one unit which uses a single transducer to transmit and receive.

If the communication were one-way, the direction of data flow would depend upon the architecture of the location system. In the case of a centralized system with mobile transmitters, someone might want to send their appointments (stored on their PDA) to a central server upon entering the office at the beginning of the working day. Or, considering the case of an anonymous, private system with mobile receivers, users might wish to receive information of general interest, such as news or weather reports.

\section{Conclusions}

In this paper, we have presented a novel polled location system. It employs Dolphin transmitters and receivers and spread spectrum signalling to allow simultaneous multiple access and to provide excellent performance in the presence of noise. Thus, the broadband ultrasonic location system has significant advantages over the narrowband systems previously developed.

The Dolphin transmitter and receiver prototype hardware were described in detail, and the above-noise bandwidth of the channel between them was shown to be approximately $75 \mathrm{kHz}$ at one metre. The prototype design can be modified for large-scale deployment by employing small, low-power components more appropriate for mobile and ubiquitous devices.

The Dolphin units were installed as a polled, centralized location system in an office, and extensive experiments were conducted. The broadband location system was shown to perform well in the presence of noise which would have caused previous narrowband systems to fail. Its potential for improved update rates through simultaneous multiple access was proven. Even with multiple units transmitting simultaneously in the presence of noise, the accuracy of the location estimates was not drastically affected, although the algorithm did return fewer readings. Ninety-five percent of the returned location estimates have an accuracy of just over two centimetres.

\section{Acknowledgements}

Mike Hazas would like to thank his supervisor, Andy Hopper, for his continuing support, and members of the Laboratory for Communications Engineering for their thoughtful discussions and helpful comments on this paper. Mike is especially grateful to Frank Hoffmann and James Scott for their assistance in hammering out the important issues during the early stages of the Dolphin project.

This material is based upon work supported under a United States National Science Foundation Graduate Fellowship.

\section{References}

[1] Gregory D. Abowd and Elizabeth D. Mynatt. Charting past, present, and future research in ubiquitous computing. ACM Transactions on Computer-Human Interaction, 7(1):29-58, March 2000. 
[2] Roy Want, Andy Hopper, Veronica Falcao, and Jon Gibbons. The Active Badge location system. ACM Transactions on Information Systems, 10(1):91-102, January 1992.

[3] Bill N. Schilit, Norman Adams, Rich Gold, Michael Tso, and Roy Want. The ParcTab mobile computing system. In Proceedings of the Fourth Workshop on Workstation Operating Systems, pages 34-39, Napa, California, USA, October 1993.

[4] D. Kirsch and T. Starner. The Locust Swarm: An environmentally-powered, networkless location and messaging system. In Proceedings of the First International Symposium on Wearable Computers, Boston, Massachusetts, USA, October 1997.

[5] Christopher Wren, Ali Azarbayejani, Trevor Darrell, and Alex Pentland. Pfinder: Realtime tracking of the human body. IEEE Transactions on Pattern Analysis and Machine Intelligence, 19(7):780-785, July 1997.

[6] Diego López de Ipiña. Video-based sensing for wide deployment of sentient spaces. In Proceedings of the Second PACT 2001 Workshop on Ubiquitous Computing and Communications, Barcelona, Spain, September 2001. ACM, IEEE.

[7] Eric Foxlin, Michael Harrington, and George Pfeifer. Constellation: A wide-range wireless motion-tracking system for augmented reality and virtual set applications. In Proceedings of the $25^{\text {th }}$ Annual Conference on Computer Graphics, pages 371-378, Orlando, Florida, USA, July 1998.

[8] Andy Ward, Alan Jones, and Andy Hopper. A new location technique for the active office. IEEE Personal Communications, 4(5):42-47, October 1997.

[9] Nissanka B. Priyantha, Anit Chakraborty, and Hari Balakrishnan. The Cricket locationsupport system. In Proceedings of the Sixth International Conference on Mobile Computing and Networking (ACM MobiCom), Boston, Massachusetts, USA, August 2000.

[10] Paramvir Bahl and Venkata N. Padmanabhan. RADAR: An in-building RF-based user location and tracking system. In Proceedings of IEEE Conference on Computer Communications (INFOCOM), volume 2, pages 775-784, Tel-Aviv, Israel, March 2000.

[11] Paul Castro, Patrick Chiu, Ted Kremenek, and Richard Muntz. A probabilistic room location service for wireless networked environments. In Proceedings of Ubicomp 2001: Ubiquitous Computing, pages 18-34, Atlanta, Georgia, USA, September 2001. ACM, SpringerVerlag.

[12] Andy Harter, Andy Hopper, Pete Steggles, Andy Ward, and Paul Webster. The anatomy of a context-aware application. In Proceedings of the Fifth International Conference on Mobile Computing and Networking (MobiCom), Seattle, Washington, USA, August 1999.

[13] Mike Addlesee, Rupert Curwen, Steve Hodges, Joe Newman, Pete Steggles, Andy Ward, and Andy Hopper. Implementing a sentient computing system. IEEE Computer, 34(8):5056, August 2001.

[14] Andy Ward. Sensor-driven Computing. PhD thesis, University of Cambridge, United Kingdom, August 1998.

[15] Nissanka B. Priyantha, Allen K. L. Miu, Hari Balakrishnan, and Seth Teller. The Cricket Compass for context-aware mobile applications. In Proceedings of the Seventh International Conference on Mobile Computing and Networking (ACM MobiCom), Rome, Italy, July 2001.

[16] Cliff Randell and Henk Muller. Low cost indoor positioning system. In Proceedings of Ubicomp 2001: Ubiquitous Computing, pages 42-48, Atlanta, Georgia, USA, September 2001. ACM, Springer-Verlag.

[17] H. E. Bass, L. C. Sutherland, A. J. Zuckerwar, D. T. Blackstock, and D. M. Hester. Atmospheric absorption of sound: Further developments. Journal of the Acoustic Society of America, 97(1):680-683, January 1995.

[18] Heiji Kawai. The piezoelectricity of poly(vinylidene fluoride). Japanese Journal of Applied Physics, 8:975-6, 1969. 
[19] H. R. Gallantree. Ultrasonic applications of PVDF transducers. The Marconi Review, 45(224):49-64, 1982.

[20] M. Platte. PVDF ultrasonic transducers. Ferroelectrics, 75(3):327-337, 1987.

[21] A. Ambrosy and K. Holdik. Piezoelectric PVDF films as ultrasonic transducers. Journal of Physics E: Scientific Instruments, 17(10):856-859, 1984.

[22] S. Pangraz and W. Arnold. Bandwidth of inhomogeneously polarized PVDF-films and their use in the design of efficient ultrasonic transducers. Ferroelectrics, 93:251-257, 1989.

[23] Antonino S. Fiorillo. Design and characterization of a PVDF ultrasonic range sensor. IEEE Trans. on Ultrasonics, Ferroelectrics, and Frequency Control, 39(6):688-692, November 1992.

[24] Antonino S. Fiorillo. PVDF ultrasonic sensors for location of small objects. Sensors and Actuators A - Physical, 42(1-3):406-409, 1994.

[25] Hong Wang and Minoru Toda. Curved PVDF airborne transducer. IEEE Trans. on Ultrasonics, Ferroelectrics, and Frequency Control, 46(6):1375-1386, November 1999.

[26] Andrew J. Viterbi. Very low rate convolutional codes for maximum theoretical performance of spread-spectrum multiple-access channels. IEEE Journal on Selected Areas in Communications, 8(4):641-649, May 1990.

[27] R. Gold. Optimal binary sequences for spread spectrum multiplexing. IEEE Trans. on Information Theory, IT-13(4):619-621, 1967.

[28] Lewis Girod and Deborah Estrin. Robust range estimation using acoustic and multimodal sensing. In IEEE/RSJ International Conference on Intelligent Robots and Systems (IROS 2001), October 2001. 